\title{
Ten easy lessons for good communication of LCA
}

\author{
Reinout Heijungs
}

Received: 8 October 2013 / Accepted: 9 October 2013 / Published online: 22 October 2013

(C) Springer-Verlag Berlin Heidelberg 2013

Some time ago, I included in an exam for my M.Sc. students a small experiment. It consisted of pasting a small fragment, just one graph, from a recent peer-reviewed article that was published in The International Journal of Life Cycle Assessment. The question to the students was easy; "Point out three mistakes." All students were able to mention two or three errors. This triggered some reflection. Are my students, who have not yet earned a M.Sc., let alone a Ph.D., more clever than a whole cluster of authors, peer-reviewers, and editors, many of whom are doctors and professors? I think I now know the answer. All these authors and peer-reviewers are clever as well, but they have become accustomed to mistakes that are so often made that they are no longer seen as wrong. My students are fresher, and easily find them.

Interaction with fresh students keeps a scientist fresh as well. Many of my heterodox points of view originate from interaction with students, who ask naive questions that are exactly to the point. It is the purpose of this paper to communicate some of my lessons learnt.

I will concentrate on communicating life cycle assessment (LCA), not on doing LCA. After all, the students read communicated results. Moreover, I will not mention issues of style, like "avoid long sentences." Obviously, scientists make many errors, in their calculations, in their argumentation, and in their English. But these are errors that anyone makes from time to time, and that reviewers and editors may easily discover. But they also make mistakes that the community as

\footnotetext{
R. Heijungs $(\bowtie)$

Institute of Environmental Sciences (CML), Leiden University, P.O. Box 9518, 2300 RA Leiden, The Netherlands e-mail: heijungs@cml.leidenuniv.nl

R. Heijungs

e-mail: r.heijungs@vu.nl

R. Heijungs

Department of Econometrics and Operations Research, VU University Amsterdam, De Boelelaan 1105, 1081 HV Amsterdam, The Netherlands
}

a whole makes. And the community will not recognize it. It is the newbie who questions the obvious.

\section{Distinguish between system expansion and substitution}

I will not state my opinion on which approach to allocation is good or wrong. I merely indicate that the literature is quoted incorrectly by so many articles, that I also make the mistake from time to time. ISO 14041/14044 mentions a preference hierarchy for dealing with multi-functional processes. One of the options is by "expanding the product system to include the additional functions related to the co-products." It seems logical to refer to this as "system expansion." But read carefully: in system expansion, you include the function of the co-products. This means that your LCA will no longer be about the original product, but about the original product plus a co-product. The functional unit is therefore enlarged with the co-product. If the LCA starts with functional unit " $1-\mathrm{kWh}$ electricity," system expansion may change it into " $1-\mathrm{kWh}$ electricity plus 2-MJ heat." It has been shown long ago that adding a function to a system is in some way "equivalent" to subtracting this function from the system. "System expansion" is thus "equivalent" to another procedure, which is known under various names, such as the "substitution method," the "avoided burden method," etc. But, "equivalent" is not the same as "equal." ISO 14041/14044 nowhere mentions terms like "avoided," "substitution," or "subtraction." It doesn't mention it, and it certainly doesn't recommend it. Nevertheless, we can find numerous articles which use the substitution method, claiming that ISO would recommend it. And we find another large group of articles which correctly claim that ISO recommends "system expansion," but then do a substitution method, putting the label of system expansion on it. Of course, you may well disagree with ISO, or anyhow dislike a standard, and choose to apply substitution. But then, please write that this differs from ISO rather than conforming to ISO. 


\section{Pay attention to flow diagrams}

Flow diagrams are a convenient way to display a product system. But they should be drawn carefully. In articles, we often see a mix of products and processes. Unfortunately, ISO doesn't provide any guidelines here. Basically, any convention will work, as long as it is used systematically. My personal guidelines are as follows:

- Processes are represented by a box;

- Products (including services and waste) are represented by arrows between boxes;

- A main direction must be chosen, e.g., from top to bottom or from left to right, although some loops may be present;

- Environmental interventions are not shown, because the diagram focuses on the structure of the processes;

- Numbers are not shown (for the same reason).

A complication that should be mentioned is that some databases and/or software do not consistently differentiate the name of a process and the name of a product. The processes in ecoinvent $\mathrm{v} 2$, for instance, have names such "operation, freight train" and "lorry $16 \mathrm{t}$ ". Visualizing a flow diagram with such information will automatically produce an inconsistent labeling. Fortunately, ecoinvent $\mathrm{v} 3$ has fixed this.

\section{Use technical terms correctly}

Science has developed its own vocabulary, with specific terms and meanings. Unfortunately, there are differences and inconsistencies in vocabulary between different scientific disciplines, and between science and daily language as well. Confusion cannot always be avoided. For example, LCA traditionally uses the term allocation in a way that is different from the way economists use it. I have even seen a text from an economist wherein it is stated that LCA ignores allocation, while allocation is, of course, one of the most contested topics in LCA. But the author was correct; LCA ignores allocation understood in an economist's meaning. In some cases, we can do better, and employ technical terms in a more correct way. An example is the term "significant." Many LCA studies conclude that product A "is significantly better" than product B. In most of these cases, the word "significantly" is merely a synonym to "much." A "significant difference" is then just another way of expressing a "big difference." But, in many disciplines (like psychology and medical science) the word "significance" points to a very specific technical notion. A difference is said to be significant whenever a statistical test is passed at a pre-defined level. The test can be a Student $t$ test, an ANOVA, etc., and the level can be 5, $1 \%$, etc., pointing to the accepted error of the test. So, there are many options, but invariably, significance means that it is the outcome of a statistical test. Interestingly, a significant difference can be quite small, and a big difference can be insignificant. So, it is not only imprecise to use the term significant without a statistical test, it is even wrong. Another upcoming example is the term "meta-analysis", which is gaining popularity in LCA. Meta-analysis has a well-defined meaning: the statistical processing of individual studies to generalize to higher level, using a well-defined theory. Most contributions on metaanalysis in LCA do not employ the theoretical concepts of the established theory on meta-analysis.

\section{LCA implies a life cycle}

One current trend in LCA research is developing highly sophisticated characterization models. Especially in an era where GIS-data and software is easily available, hyperregionalized approaches are flourishing. But there is a danger in this. The fallacious logic is as follows: (1) in LCA you need characterization factors; (2a) calculating characterization factors is doing LCA, or (2b) using characterization factors is doing LCA. Some of the methods developed are now illustrated on something that has no life cycle, e.g., on an emission of a chemical. And some people use characterization factors outside LCA (which is not incorrect), and then claim that they're doing LCA (which is incorrect). The error is explainable. Whenever you want to aggregate several environmental stressors, you easily run into the concept of characterization factors. Now, when you search the internet for characterization factors, you find USEtox, $\mathrm{ReCiPe}$, etc. These indeed provide long lists of characterization factors, but they also have "LCIA" or "LCA" or "life cycle" in large print on their website. A naive user may thus think that using such systems is the same as doing a life cycle study.

\section{LCA is not the same as sustainability analysis}

In the past decade, the emphasis in environmental management has broadened to a sustainability point of view. Products should not only be environmentally friendly, but they should also be economically good (eco-efficient), and issues like fair trade have spawned the interest in the social side of products as well. The triple bottom line, with PeoplePlanet-Profit (or Prosperity instead of Profit), and the idea of the co-existence of Society, Ecology, and Economy have gained popularity. This shift has been felt at many places, but it was not always a deep shift; sometimes it was more cosmetic. For instance, the book "Blueprint for a green economy" has been reissued as "Blueprint for a sustainable economy," not essentially changing the message, but seizing the new opportunity. The same can be seen in LCA. LCA is 
essentially an environmental information tool. Nevertheless, some authors use LCA to develop "sustainable products." Doing LCA and referring to the results as supporting sustainable policies is misleading. Fortunately, the LCA community is now trying to develop a life cycle sustainability analysis, in which LCA has a place together with the appropriate tools for economic and social assessment. Still, even with the other two pillars added, it is questionable if such an analysis deserves the tile of sustainability as long as the planetary boundaries, total consumption levels, and other macro features that are decisive for a sustainable world are not included. Cars can be very sustainable, as long as there are just a few of them. The problem starts when you have millions or billions of cars. So, how can an LCA ever claim to be devoted to "sustainable cars?"

\section{Do not invent units}

Quite some years ago, I wrote an editorial in this journal on the use of units in LCA. The effect has been small so far. Despite the provision in the guide for authors to use SI-units, many articles in this journal still use weird units, such as "DALY (Disability-Adjusted Life Year)," "milli-point (mPt)," and "Potentially affected fraction of species (PAF)." Of course, the SI is not complete; for instance, it lacks monetary units such as dollar or yen. So from time to time, it may be needed to introduce additional units outside SI. But for the given examples, this is not needed. "DALY" is just a year, and the other two are dimensionless.

\section{Distinguish impact from potential}

In the same editorial, I raised the issue of the names of the results of characterization. Also, my message here has been very ineffective so far. There are so many articles that claim to calculate a "Global Warming Potential (GWP)" or a "Photochemical Ozone Creation Potential (POCP)" that I sometimes start to believe to be wrong myself. The truth is: GWPs are a series of numbers that have been calculated and published by IPCC. In LCA, you may use them, by multiplying the appropriate inventory results with the corresponding GWPs. The result is an impact indicator of global warming, climate change, greenhouse effect, or however you call it. We may refer to this result in various ways, e.g., "global warming score," "climate change indicator," "greenhouse effect result." My personal preferences are "global warming (GW)" and "climate change (CC)." But in any case, in an LCA study, you don't calculate GWPs. Every paper that claims that "product A has a larger GWP than product B" or that "transportation makes the biggest contribution to GWP" is basically wrong and should be revised by the authors.

\section{Conformity to ISO or ILCD is difficult}

Many LCA studies claim that ISO was used, or an ISOconform LCA was done. Indeed, superficially, it looks so: there is a phasing into goal and scope definition, inventory analysis, etc., there is a functional unit and then there is a discussion on how allocation was done. But being ISO-compliant means a lot more! For instance, clause 4.3.2.1 states that "To decrease the risk of misunderstandings..., a description of each unit process shall be recorded." That can easily take 100 pages. And consider clause 4.4.2.1: "The LCIA phase shall include the following mandatory elements: ... classification". Did you ever see an LCA study reporting the mandatory classification? The same applies - and even more so - to the EU's International Reference Life Cycle Data System (ILCD) guidelines. It is perfectly understandable that no one can achieve $100 \%$ compliance with ISO or ILCD, and certainly not within a limited time, budget, and word count. But then, don't claim it is.

\section{Pay attention to graphs}

Many scientists have taken a course in "scientific writing," but few take one in "scientific visualization." In the old days, this was not a problem. A scientist sent a hand-made graph to the graphical service of the university, and a professional designer made some nice "art work" from this. These days, making graphs is easy and available to everyone. Scientific articles contain more graphs than ever. But this doesn't mean they are good. I recently had a pleasant day with just three recent issues of The International Journal of Life Cycle Assessment, selecting "bad graphs" for my students. This was in fact embarrassing: a peer-reviewed journal with a good impact factor, and then so many examples of bad practice. Roughly speaking, we can distinguish the following types of issues:

- Bad use of colors or shading. In pie charts or bar charts, different colors or different shading patterns are used. But the colors are often too similar (or converted to gray), and the segments shown are often too small to display the patterns. This applies also to the legend.

- Bad use of 3D. If you show a pie chart in 3D, some segments will be shown more nearby than others, and the projection may distort the sizes.

- Too brief captions. Figure captions should explain the different features. A caption like "Results of the LCA" is uninformative.

- Too much information. A figure should convey the essential information at one glance. This implies that you should not put in redundant information. What sense does it make to add to a pie chart the percentages, up to three digits? 


\section{Cite correctly}

Incorrect citing and quoting is an example of a self-enforcing phenomenon. If you read ten papers, all of which refer to the authors "Canals" and "Haes", you will start to believe that this is the correct name. It is not always so easy to check that this is not so. If the original paper has the name "Llorenç Milà $\mathrm{i}$ Canals" or "Helias Udo de Haes," it may be difficult to understand where the given names end and the surname starts. Not only are personal names incorrectly cited. Brand names are also misspelled. The products from "ecoinvent" are often cited with one capital "Ecoinvent" or even two capitals "EcoInvent". The same applies to "SimaPro," "GaBi," etc. Citing the appropriate sources is also difficult. I have frequently met people who were surprised to learn that "CML" is an institute rather than a method. Other people claim to use "the CML method", which is again unclear: CML has made many contributions to methods, from allocation to weighting, and even outside LCA, so what are they referring to? Already better, but still wrong, is "CML2001". Yes, there was a draft from CML online in 2001, but the final version was published in 2002. Did all these people use the draft method instead of the final one? Or did they forget to update the reference? Or did they rely on software, which, correctly or incorrectly, used this term? People at CML are humble enough to never refer to "the CML method", just like Planck never spoke about "Plancks's constant." At CML, we refer to the document, such as "Guinée et al. 2002" or the method's name, in this case "CML-IA". Finally, quoting material turns out to be difficult as well. One example may suffice. Many articles mention that ISO 14040's framework has a "goal definition" or a "goal and scope phase". It doesn't: it has a "goal and scope definition" phase. Occasionally, we even see articles quoting ISO 14040 with an "improvement analysis." There is not anything in ISO 14040 which even resembles that; it is just false memory of the older documents from SETAC.

Acknowledgments Students from the M.Sc. programme Industrial Ecology, jointly organized by Leiden University and Technical University Delft enriched my critical view. The same applies to students from the Leiden University College, a liberal science and arts B.Sc. residential programme in The Hague. My colleague Jeroen Guinée gave useful comments to a draft. 